\title{
Free-living marine nematodes from San Antonio Bay (Río Negro, Argentina)
}

\author{
Gabriela Villares', Virginia Lo Russo', Catalina Pastor de Ward', \\ Viviana Milano' ${ }^{2}$ Lidia Miyashiro ${ }^{3}$, Renato Mazzanti ${ }^{3}$ \\ I Laboratorio de Meiobentos LAMEIMA-CENPAT-CONICET, Boulevard Brown 2915, U9120ACF, Puerto \\ Madryn, Argentina 2 Universidad Nacional de la Patagonia San Juan Bosco, sede Puerto Madryn. Boulevard \\ Brown 3051, U9120ACF, Puerto Madryn, Argentina 3 Centro de Cómputos CENPAT-CONICET, Boulevard \\ Brown 2915, U9120ACF, Puerto Madryn, Argentina \\ Corresponding author: Gabriela Villares (villares@cenpat-conicet.gob.ar)
}

Academic editor: H-P Fagerholm | Received 18 November 2015 | Accepted 11 February 2016 | Published 28 March 2016

http://zoobank.org/3E8B6DD5-51FA-499D-AA94-6D426D5B1913

Citation: Villares G, Lo Russo V, Pastor de Ward C, Milano V, Miyashiro L, Mazzanti R (2016) Free-living marine nematodes from San Antonio Bay (Río Negro, Argentina). ZooKeys 574: 43-55. doi: 10.3897/zookeys.574.7222

\begin{abstract}
The dataset of free-living marine nematodes of San Antonio Bay is based on sediment samples collected in February 2009 during doctoral theses funded by CONICET grants. A total of 36 samples has been taken at three locations in the San Antonio Bay, Santa Cruz Province, Argentina on the coastal littoral at three tidal levels. This presents a unique and important collection for benthic biodiversity assessment of Patagonian nematodes as this area remains one of the least known regions. In total 7,743 specimens of free-living marine nematodes belonging to two classes, eight orders, 37 families, 94 genera and 104 species were collected.
\end{abstract}

\section{Keywords}

Nematoda, Enoplea, Chromadorea, South Atlantic

Copyright Gabriela Villares et al. This is an open access article distributed under the terms of the Creative Commons Attribution License (CC BY 4.0), which permits unrestricted use, distribution, and reproduction in any medium, provided the original author and source are credited. 


\section{Introduction}

This is the first study on nematodes performed on a sub-Antarctic salt marsh along the coast of Río Negro Province, Argentina. This site has a high biodiversity and was declared a Protected Natural Area N 2.670 of the province of Río Negro in 1993 as well as an international reserve of the hemispheric network of Shorebird Reserve within the Wetlands program for the Americas. However, it is also an urban center where economic, industrial and tourist activities take place. The objectives of the study were to collect, identify and discover the structure and diversity of the nematode community of San Antonio Bay. The coverage (Figure 1) of this dataset includes two classes: Chromadorea (76\%) and Enoplea (24\%); eight orders: Monhysterida (39\%), followed by Enoplida (23\%) and Chromadorida (19\%) as main occurrences; and thirty-seven families (see Figure 1).

\section{Taxonomic ranks}

Kingdom: Animalia

Phylum: Nematoda

Class: Chromadorea, Enoplea

Order: Monhysterida, Enoplida, Chromadorida, Desmodorida, Araeolaimida, Plectida, Rhabditida, Dorylaimida.

Family: Xyalidae, Linhomoeidae, Monhysteridae, Sphaerolaimidae, Siphonolaimidae, Chromadoridae, Cyatholaimidae, Ethmolaimidae, Selachinematidae, Microlaimidae, Desmodoridae, Monoposthiidae, Coninckiidae, Comesomatidae, Diplopeltidae, Axonolaimidae, Leptolaimidae, Aegialoalaimidae, Haliplectidae, Ceramonematidae, Tarvaiidae, Tylenchidae, Mesorhabditidae, Criconematidae, Panagrolaimidae, Oncholaimidae, Oxystominidae, Ironoidae, Thoracostomopsidae, Tripyloididae, Anticomidae, Anoplostomatidae, Enchelidiidae, Trefusiidae, Lauratonematidae, Enoplidae, Dorylaimidae.

Genera: Odontophora, Synodontium, Comesoma, Metasabatieria, Sabatieria, Coninckia, Campylaimus, Diplopeltula, Chromadora, Chromadorella, Chromadorina, Prochromadorella, Actinonema, Rhips, Dichromadora, Neochromadora, Spilophorella, Marylynnia, Paracantonchus, Paracyatholaimus, Pomponema, Paraethmolaimus, Gammanema, Halichoanolaimus, Latronema, Molgolaimus, Metachromadora, Onyx, Polysigma, Spirinia, Bolbolaimus, Microlaimus, Nudora, Desmolaimus, Metalinhomoeus, Terschellingia, Eleutherolaimus, Paralinhomoeus, Siphonolaimus, Diplolaimella, Diplolaimelloides, Halomonhystera, Monhystera, Sphaerolaimus, Amphimonhystera, Cobbia, Daptonema, Gonionchus, Linhystera, Metadesmolaimus, Omicronema, Paramonohystera, Promonhystera, Pseudosteineria, Rhynchonema, Theristus, Xyalidae gen.1, Ceramonema, Pselionema, Tarvaia, Haliplectus, Cyartonema, Deontolaimus, Leptolaimus, Mesorhabditis, Macroposthonia, Panagrolaimus, Tylenchus, Dorylaimus, Chaetonema, Cephalanticoma, Enoplus, Epacanthion, Oxyonchus, Thoracostomopsidae gen.1, Conilia, Dolicholaimus, Syringolaimus, Halalaimus, Calyptronema, Eu- 


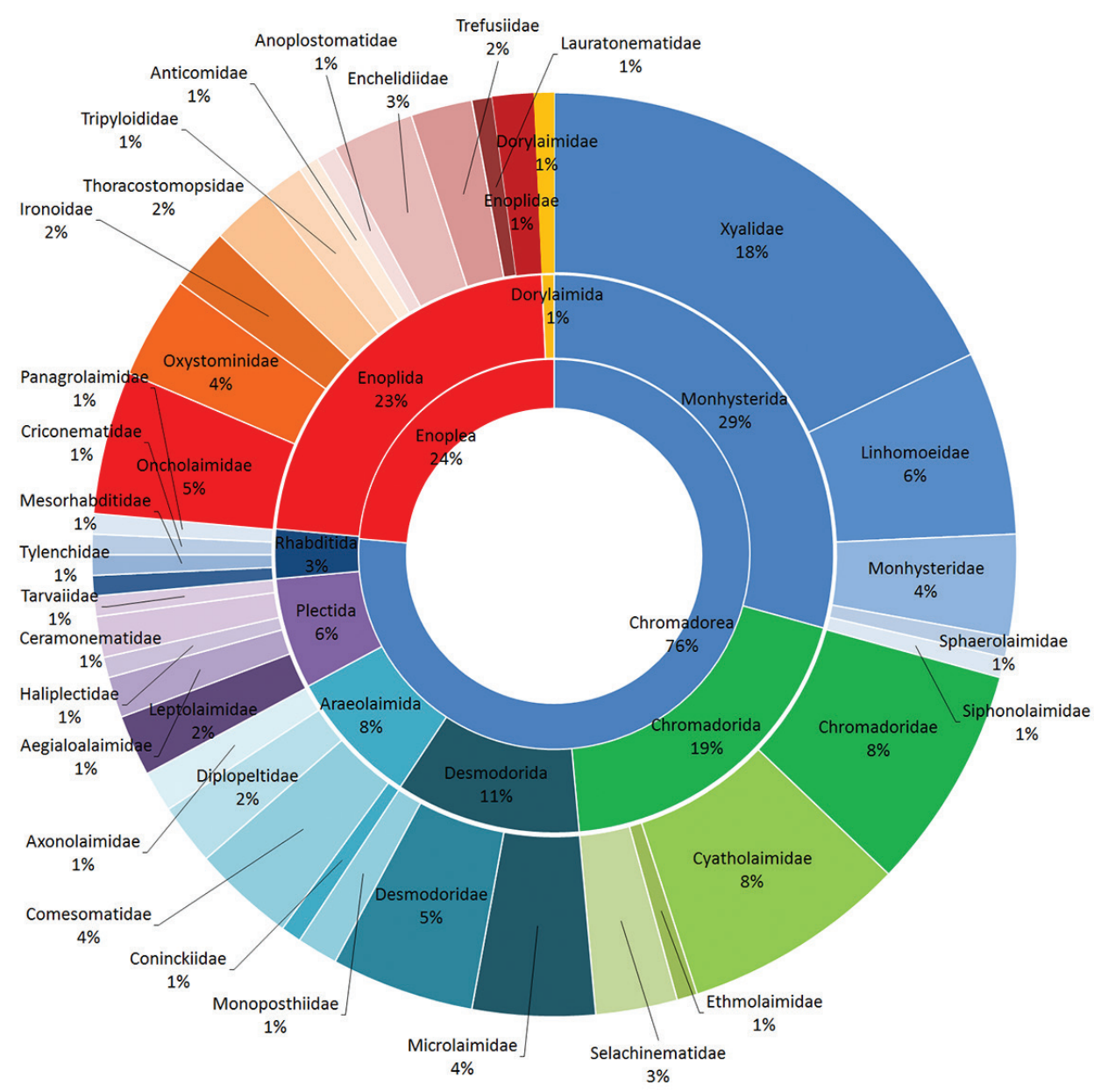

Figure I. Taxonomic coverage by class, order and family.

rystomina, Abelbolla, Adoncholaimus, Viscosia, Metoncholaimus, Oncholaimus, Lauratonema, Rhabdocoma, Trefusia, Trefusiidae gen.1, Bathylaimus, Tripyloides.

Species with higher occurrences: Microlaimus globiceps, Paraethmolaimus dahli, Thalassomonhystera parva, Microlaimus decoratus, Diplolaimelloides oschei, Nudora crepidata, Viscosia macramphida, Chromadorina longispiculum, Diplolaimella gerlachi, Leptolaimus luridus.

\section{Spatial coverage}

General spatial coverage: San Antonio Bay, Río Negro Province, Argentina (Figure 2). For this study three sites were selected: "Ciudad" (A), located in the north of the bay; "Baliza Camino" (B), located off the mouth of the bay of San Antonio and "Banco Perdices" (C), 


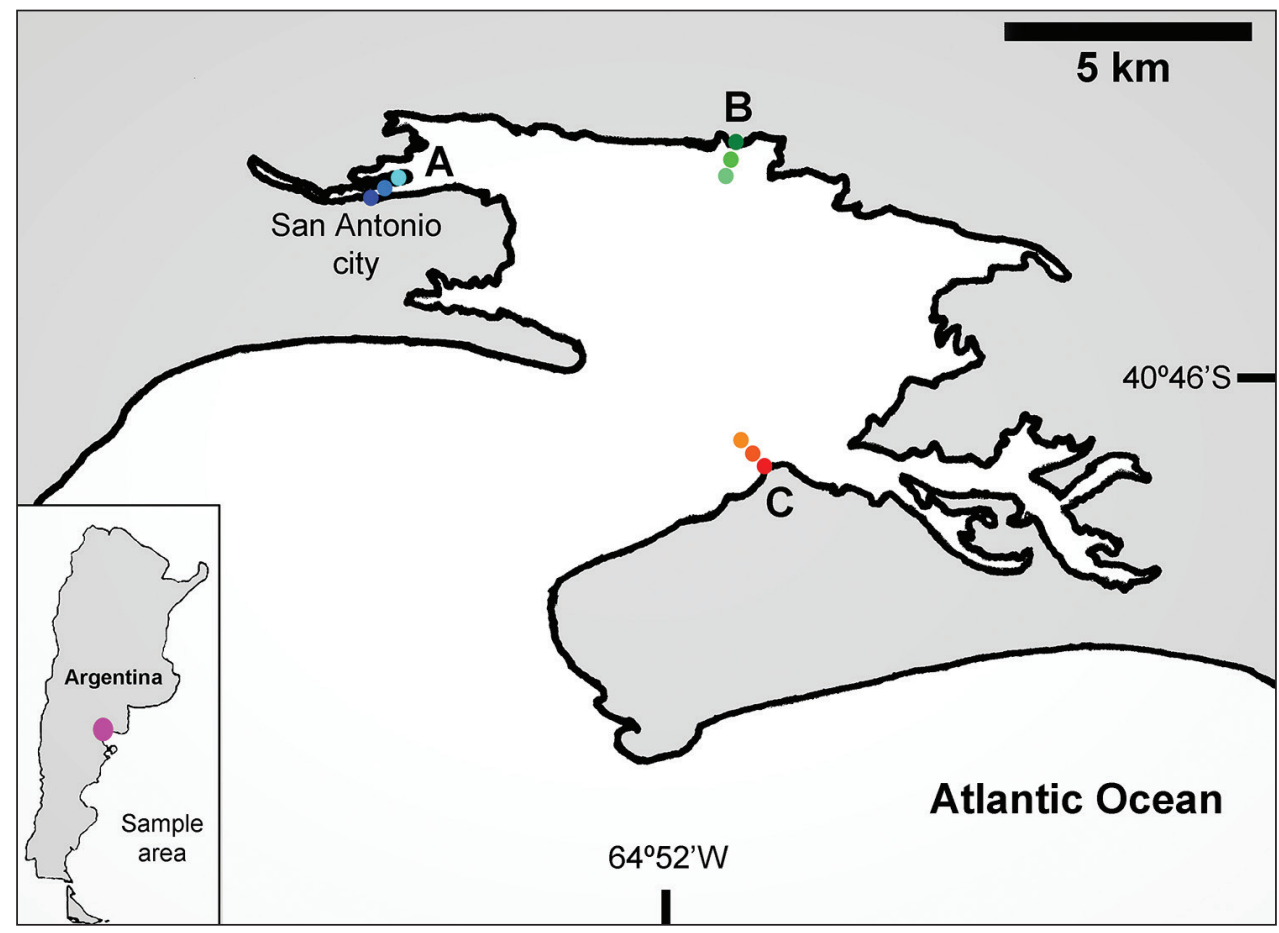

Figure 2. Spatial coverage. San Antonio Bay, Argentina. Sites: A "Ciudad" B "Baliza Camino" C "Banco Perdices". Levels $=\mathrm{u}, \mathrm{m}, 1$..

located $12 \mathrm{~km}$ south of "Las Grutas". At each sampling site, three tidal levels were chosen: upper-littoral, high tide, salt-marsh habitat (u); middle littoral, mean tide, un-vegetated habitat $(\mathrm{m})$ and low littoral, low tide, un-vegetated habitat (l) (Figure 3).

Coordinates: "Ciudad": $\mathrm{Au}=40^{\circ} 43^{\prime} 40.2^{\prime \prime} \mathrm{S} ; 64^{\circ} 57^{\prime} 41.1^{\prime W} \mathrm{~W} ; \mathrm{Am}=40^{\circ} 43^{\prime} 39.0^{\prime \prime S}$;

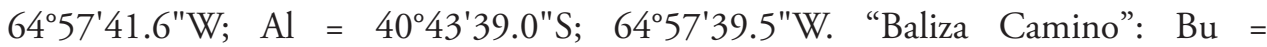
$40^{\circ} 42^{\prime} 59.9^{\prime \prime} \mathrm{S} ; 64^{\circ} 50^{\prime} 46.8^{\prime \prime} \mathrm{W} ; \mathrm{Bm}=40^{\circ} 43^{\prime} 05.8^{\prime \prime} \mathrm{S} ; 64^{\circ} 50^{\prime} 58.5^{\prime \prime} \mathrm{W} ; \mathrm{Bl}=40^{\circ} 43^{\prime} 11.6^{\prime \prime} \mathrm{S}$; 64051'14,6"W. "Banco Perdices": $\mathrm{Cu}=40^{\circ} 47^{\prime} 00.8^{\prime \prime S} ; 64^{\circ} 50^{\prime} 54.3^{\prime \prime W} ; \mathrm{Cm}=$ $40^{\circ} 47^{\prime} 05.6^{\prime \prime S}$; 6451'17.8"W; $\mathrm{Cl}=40^{\circ} 46^{\prime} 51.9^{\prime \prime} \mathrm{S} ; 64^{\circ} 51^{\prime} 02.8^{\prime \prime W}$.

\section{Temporal coverage}

12-14 February 2009.

\section{Methods}

Sampling description: At each site and level location, four replicates $(20 \mathrm{ml})$ were sampled with a PVC syringe $(60 \mathrm{ml}$, inner diameter $2.9 \mathrm{~cm})$ and separated by a dis- 

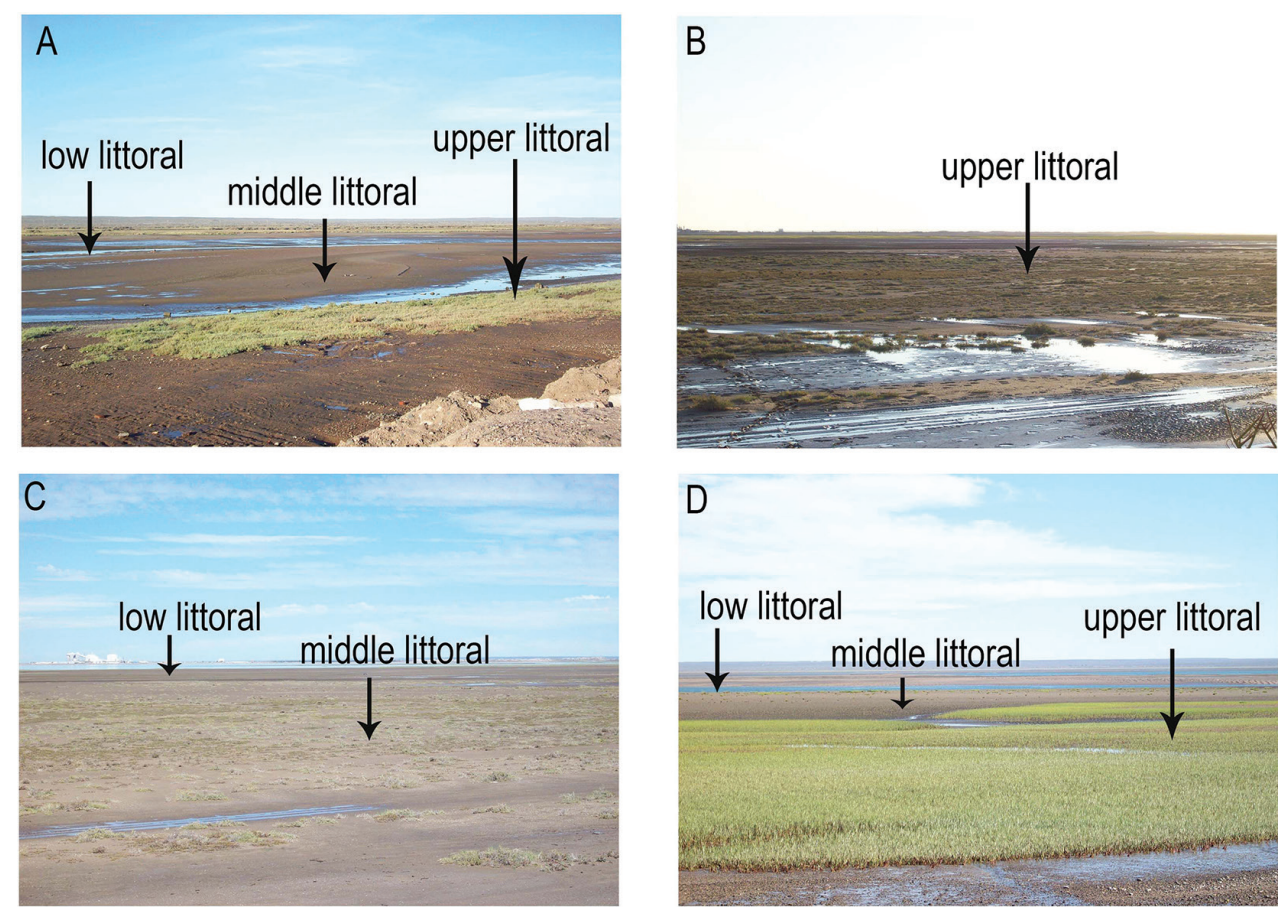

Figure 3. San Antonio Bay, Argentina. Views from the sampling sites. A "Ciudad" B, C "Baliza Camino" D "Banco Perdices".

tance of 5-10 m each: four for marine nematodes counts, two for organic matter and two for sediment analyses. Each sample was fixed in situ, with a solution of 5\% formaldehyde in filtered sea water with the addition of Rose Bengal tint. Marine nematodes were extracted from samples using the elutriation/decantation LUDOX TM (colloidal silica polymer) method at a specific gravity of 1.15 , quantifying only organisms passing through a $500 \mu \mathrm{m}$ mesh and then retained by a $63 \mu \mathrm{m}$ mesh. Samples were evaporated to anhydrous glycerol and permanent slides made (Somerfield and Warwick 1996). The taxonomic classification followed proposed by De Ley and Blaxter (2004). For the identification of species international keys (Platt and Warwick 1983, Platt and Warwick 1988, Warwick et al. 1998, Lorenzen 1994, Abebe et al. 2006) and taxonomical papers (Pastor de Ward 1978, 1980, 1984a, b, c, d, e, 1985, 1986, 1988, 1989, 1990, 1991, 1993, 1995a, b, 1996, 1998a, b, c, 1999, Pastor de Ward and Lo Russo 2009, Villares and Pastor de Ward 2012, Lo Russo et. al. 2012, Villares et al. 2013, Pastor de Ward et al. 2013, Lo Russo et. al. 2015) were used. Holotypes and paratypes are deposited in the Collection of Nematodes of the Centro Nacional Patagónico (CENPAT-CONICET), Chubut, Argentina recognized as National Service of Biological Data of Argentina and included in their web page from 2011 (http:// www.gbif.org/dataset/06df03fc-8973-490c-af74-089fffae9e24; http://www.gbif.org/ dataset/d592283b-b00e-4a39-9499-289842ccddf1). 


\section{Project details}

Project title: "Comparación de comunidades de nematodos de marismas de San Antonio Oeste (río Negro) y San Julián (Sta. Cruz)". [Comparison of nematode marsh communities of San Antonio Oeste (Río Negro) and San Julián (Sta. Cruz)]. Doctoral thesis Universidad Nacional del Comahue (Lo Russo 2012).

"Diversidad funcional y producción secundaria de las comunidades de nematodos de las marismas de San Antonio (Rio Negro) y de la ría de San Julián (Santa Cruz)". [Functional diversity and secondary production of nematode marsh communities of San Antonio (Río Negro) estuary and San Julián (Santa Cruz)". Doctoral thesis Universidad Nacional del Comahue (Villares 2014).

Personnel: Catalina Pastor de Ward (Project Director, meio-benthos specialist), Virginia Lo Russo and Gabriela Villares (field work, nematodes identification, data collection and analysis), Viviana Milano (grant-holding student, data input), Lidia Miyashiro (Darwin core data input), Renato Mazzanti (software engineer, data base manager).

Study extent description: The San Antonio Bay marine nematodes is a dataset that gives new insights on the taxonomic and geographic distribution of south Atlantic marine nematodes, covering an under-explored region of the southern Atlantic coasts. This is the first study on marine nematodes in this site. This dataset presents species occurrences and species richness of the individual free-living marine nematodes present at three coastal areas ("Ciudad", "Baliza Camino", "Banco Perdices") of the San Antonio Bay at three different tidal levels (upper, middle and low-littoral).

In total 7,443 specimens of free-living marine nematodes belonging to two classes, eight orders, 37 families, 94 genera and 140 species were collected.

\begin{tabular}{l|l|l|l}
\hline Genera and Species & Family & Order & Class \\
\hline Odontophora peritricha Wieser, 1956 & Axonolaimidae & Araeolaimida & Chromadorea \\
\hline Synodontium sp. 1 & Axonolaimidae & Araeolaimida & Chromadorea \\
\hline Comesoma sp. 1 & Comesomatidae & Araeolaimida & Chromadorea \\
\hline Metasabatieria sp. 1 & Comesomatidae & Araeolaimida & Chromadorea \\
\hline Sabatieria mortenseni (Ditlevsen, 1921) & Comesomatidae & Araeolaimida & Chromadorea \\
\hline Sabatieria punctata Kreis, 1924$)$ & Comesomatidae & Araeolaimida & Chromadorea \\
\hline Sabatieria wieseri Platt, 1985 & Comesomatidae & Araeolaimida & Chromadorea \\
\hline Coninckia sp. 1 & Coninckiidae & Araeolaimida & Chromadorea \\
\hline Campylaimus gerlachi Timm, 1961 & Diplopeltidae & Araeolaimida & Chromadorea \\
\hline Campylaimus sp. 2 & Diplopeltidae & Araeolaimida & Chromadorea \\
\hline Diplopeltula sp.1 & Diplopeltidae & Araeolaimida & Chromadorea \\
\hline Chromadora nudicapitata Bastian, 1865 & Chromadoridae & Chromadorida & Chromadorea \\
\hline Chromadorella sp.1 1 & Chromadoridae & Chromadorida & Chromadorea \\
\hline Chromadorina longispiculum Pastor de Ward, 1985 & Chromadoridae & Chromadorida & Chromadorea \\
\hline Prochromadorella sp. 1 & Chromadoridae & Chromadorida & Chromadorea \\
\hline Prochromadorella sp. 2 & Chromadoridae & Chromadorida & Chromadorea \\
\hline Actinonema sp. 1 & Chromadoridae & Chromadorida & Chromadorea \\
\hline
\end{tabular}




\begin{tabular}{|c|c|c|c|}
\hline Genera and Species & Family & Order & Class \\
\hline Rhips sp. 1 & Chromadoridae & Chromadorida & Chromadorea \\
\hline Dichromadora sp. 1 & Chromadoridae & Chromadorida & Chromadorea \\
\hline $\begin{array}{l}\text { Neochromadora alejandroi Lo Russo \& Pastor de } \\
\text { Ward, } 2012\end{array}$ & Chromadoridae & Chromadorida & Chromadorea \\
\hline Neochromadora papillosa Pastor de Ward, 1865 & Chromadoridae & Chromadorida & Chromadorea \\
\hline Spilophorella paradoxa (De Man, 1888) & Chromadoridae & Chromadorida & Chromadorea \\
\hline Marylynnia sp. 1 & Cyatholaimidae & Chromadorida & Chromadorea \\
\hline Marylynnia sp. 2 & Cyatholaimidae & Chromadorida & Chromadorea \\
\hline Marylynnia sp. 3 & Cyatholaimidae & Chromadorida & Chromadorea \\
\hline Paracanthonchus austrospectabilis Wieser, 1954 & Cyatholaimidae & Chromadorida & Chromadorea \\
\hline Paracanthonchus punctatus (Bastian, 1865) & Cyatholaimidae & Chromadorida & Chromadorea \\
\hline Paracanthonchus sp. 1 & Cyatholaimidae & Chromadorida & Chromadorea \\
\hline Paracanthonchus sp. 2 & Cyatholaimidae & Chromadorida & Chromadorea \\
\hline Paracanthonchus sp. 3 & Cyatholaimidae & Chromadorida & Chromadorea \\
\hline Paracyatholaimus chilensis Gerlach, 1953 & Cyatholaimidae & Chromadorida & Chromadorea \\
\hline Paracyatholaimus sp. 1 & Cyatholaimidae & Chromadorida & Chromadorea \\
\hline Pomponema sp. 1 & Cyatholaimidae & Chromadorida & Chromadorea \\
\hline Paraethmolaimus dabli (Gerlach, 1953) & Ethmolaimidae & Chromadorida & Chromadorea \\
\hline Gammanema sp. 1 & Selachinematidae & Chromadorida & Chromadorea \\
\hline Gammanema sp. 2 & Selachinematidae & Chromadorida & Chromadorea \\
\hline Halichoanolaimus sp. 1 & Selachinematidae & Chromadorida & Chromadorea \\
\hline Latronema sp. 1 & Selachinematidae & Chromadorida & Chromadorea \\
\hline Molgolaimus sp. 1 & Desmodoridae & Desmodorida & Chromadorea \\
\hline Molgolaimus sp. 2 & Desmodoridae & Desmodorida & Chromadorea \\
\hline Metachromadora sp. 1 & Desmodoridae & Desmodorida & Chromadorea \\
\hline Metachromadora spectans Gerlach, 1957 & Desmodoridae & Desmodorida & Chromadorea \\
\hline Onyx sp. 1 & Desmodoridae & Desmodorida & Chromadorea \\
\hline Polysigma sp. 1 & Desmodoridae & Desmodorida & Chromadorea \\
\hline Spirinia septentrionalis Cobb, 1914 & Desmodoridae & Desmodorida & Chromadorea \\
\hline Bolbolaimus sp. 2 & Microlaimidae & Desmodorida & Chromadorea \\
\hline Microlaimus conothelis (Lorenzen, 1973) Jensen, 1978 & Microlaimidae & Desmodorida & Chromadorea \\
\hline Microlaimus capillaris Gerlach, 1957 & Microlaimidae & Desmodorida & Chromadorea \\
\hline Microlaimus decoratus Pastor de Ward, 1991 & Microlaimidae & Desmodorida & Chromadorea \\
\hline Microlaimus globiceps De Man, 1880 & Microlaimidae & Desmodorida & Chromadorea \\
\hline Microlaimus sp. 2 & Microlaimidae & Desmodorida & Chromadorea \\
\hline Nudora besnardi (Gerlach, 1956) & Monoposthiidae & Desmodorida & Chromadorea \\
\hline Nudora crepidata Wieser, 1954 & Monoposthiidae & Desmodorida & Chromadorea \\
\hline Desmolaimus sp. 3 & Linhomoeidae & Monhysterida & Chromadorea \\
\hline Desmolaimus sp. 4 & Linhomoeidae & Monhysterida & Chromadorea \\
\hline Metalinhomoeus gloriae Pastor de Ward, 1989 & Linhomoeidae & Monhysterida & Chromadorea \\
\hline Metalinhomoeus parafiliformis Pastor de Ward, 1989 & Linhomoeidae & Monhysterida & Chromadorea \\
\hline Metalinhomoeus typicus De Man, 1907 & Linhomoeidae & Monhysterida & Chromadorea \\
\hline Terschellingia longicaudata De Man, 1907 & Linhomoeidae & Monhysterida & Chromadorea \\
\hline Terschellingia sp. 2 & Linhomoeidae & Monhysterida & Chromadorea \\
\hline Eleutherolaimus sp. 1 & Linhomoeidae & Monhysterida & Chromadorea \\
\hline Paralinhomoeus visitus Pastor de Ward, 1989 & Linhomoeidae & Monhysterida & Chromadorea \\
\hline
\end{tabular}




\begin{tabular}{|c|c|c|c|}
\hline Genera and Species & Family & Order & Class \\
\hline Siphonolaimus auratus Wieser, 1956 & Siphonolaimidae & Monhysterida & Chromadorea \\
\hline Diplolaimella gerlachi Pastor de Ward, 1984 & Monhysteridae & Monhysterida & Chromadorea \\
\hline Diplolaimella ocellata (Bütschli, 1874) & Monhysteridae & Monhysterida & Chromadorea \\
\hline Diplolaimelloides oschei Meyl, 1954 & Monhysteridae & Monhysterida & Chromadorea \\
\hline Halomonhystera disjuncta (Bastian, 1865) & Monhysteridae & Monhysterida & Chromadorea \\
\hline Thalassomonhystera parva (Bastian, 1865) & Monhysteridae & Monhysterida & Chromadorea \\
\hline Sphaerolaimus pacificus Allgen, 1945 & Sphaerolaimidae & Monhysterida & Chromadorea \\
\hline Amphimonhystera sp. 2 & Xyalidae & Monhysterida & Chromadorea \\
\hline Cobbia macrodentata Lo Russo \& Pastor de Ward, 2012 & Xyalidae & Monhysterida & Chromadorea \\
\hline Daptonema laxus Wieser, 1956 & Xyalidae & Monhysterida & Chromadorea \\
\hline Daptonema sp. 2 & Xyalidae & Monhysterida & Chromadorea \\
\hline Daptonema sp. 3 & Xyalidae & Monhysterida & Chromadorea \\
\hline Gonionchus sp. 1 & Xyalidae & Monhysterida & Chromadorea \\
\hline Linhystera sp. 1 & Xyalidae & Monhysterida & Chromadorea \\
\hline Metadesmolaimus sp. 3 & Xyalidae & Monhysterida & Chromadorea \\
\hline Metadesmolaimus sp. 4 & Xyalidae & Monhysterida & Chromadorea \\
\hline Metadesmolaimus sp. 5 & Xyalidae & Monhysterida & Chromadorea \\
\hline Omicronema sp. 1 & Xyalidae & Monhysterida & Chromadorea \\
\hline Paramonohystera sp. 4 & Xyalidae & Monhysterida & Chromadorea \\
\hline Promonhystera sp. 1 & Xyalidae & Monhysterida & Chromadorea \\
\hline Pseudosteineria sp. 1 & Xyalidae & Monhysterida & Chromadorea \\
\hline Rhynchonema separatum Lorenzen, 1975 & Xyalidae & Monhysterida & Chromadorea \\
\hline Rhynchonema sp. 1 & Xyalidae & Monhysterida & Chromadorea \\
\hline Rhynchonema sp. 2 & Xyalidae & Monhysterida & Chromadorea \\
\hline Rhynchonema sp. 3 & Xyalidae & Monhysterida & Chromadorea \\
\hline Theristus lorenzeni Pastor de Ward, 1985 & Xyalidae & Monhysterida & Chromadorea \\
\hline Theristus modicus Wieser, 1956 & Xyalidae & Monhysterida & Chromadorea \\
\hline Theristus sp. 2 & Xyalidae & Monhysterida & Chromadorea \\
\hline Theristus sp. 3 & Xyalidae & Monhysterida & Chromadorea \\
\hline Theristus sp. 4 & Xyalidae & Monhysterida & Chromadorea \\
\hline Theristus sp. 5 & Xyalidae & Monhysterida & Chromadorea \\
\hline Xyalidae gen. 1 sp. 1 & Xyalidae & Monhysterida & Chromadorea \\
\hline Ceramonema sp. 1 & Ceramonematidae & Plectida & Chromadorea \\
\hline Pselionema sp. 1 & Ceramonematidae & Plectida & Chromadorea \\
\hline Tarvaia sp. 1 & Tarvaiidae & Plectida & Chromadorea \\
\hline Haliplectus salicornius Pastor de Ward, 1984 & Haliplectidae & Plectida & Chromadorea \\
\hline Cyartonema flexile Cobb, 1920 & Aegialoalaimidae & Plectida & Chromadorea \\
\hline Cyartonema sp. 1 & Aegialoalaimidae & Plectida & Chromadorea \\
\hline Deontolaimus papillatus De Man, 1880 & Leptolaimidae & Plectida & Chromadorea \\
\hline Leptolaimus luridus Timm, 1963 & Leptolaimidae & Plectida & Chromadorea \\
\hline Leptolaimus puccinelliae Gerlach, 1959 & Leptolaimidae & Plectida & Chromadorea \\
\hline Mesorhabditis sp. 2 & Mesorhabditidae & Rhabditida & Chromadorea \\
\hline Macroposthonia sp. 1 & Criconematidae & Rhabditida & Chromadorea \\
\hline Panagrolaimus sp. 1 & Panagrolaimidae & Rhabditida & Chromadorea \\
\hline Tylenchus sp. 1 & Tylenchidae & Rhabditida & Chromadorea \\
\hline
\end{tabular}




\begin{tabular}{|c|c|c|c|}
\hline Genera and Species & Family & Order & Class \\
\hline Dorylaimus sp. 1 & Dorylaimidae & Dorylaimida & Enoplea \\
\hline Chaetonema patagonica Lo Russo et al., 2015 & Anoplostomatidae & Enoplida & Enoplea \\
\hline Cephalanticoma sp. 1 & Anticomidae & Enoplida & Enoplea \\
\hline Enoplus benhami Ditlevsen, 1930 & Enoplidae & Enoplida & Enoplea \\
\hline Enoplus meridionalis Steiner, 1921 & Enoplidae & Enoplida & Enoplea \\
\hline Epacanthion bicuspidatum Lo Russo et al., 2012 & Thoracostomopsidae & Enoplida & Enoplea \\
\hline Oxyonchus sp. 1 & Thoracostomopsidae & Enoplida & Enoplea \\
\hline Thoracostomopsidae gen. 1 sp. 2 & Thoracostomopsidae & Enoplida & Enoplea \\
\hline Conilia divina Gerlach, 1956 & Ironoidae & Enoplida & Enoplea \\
\hline Dolicholaimus marioni De Man, 1888 & Ironoidae & Enoplida & Enoplea \\
\hline Syringolaimus smarigdus Cobb, 1928 & Ironoidae & Enoplida & Enoplea \\
\hline Halalaimus sp. 1 & Oxystominidae & Enoplida & Enoplea \\
\hline Halalaimus sp. 2 & Oxystominidae & Enoplida & Enoplea \\
\hline Halalaimus sp. 3 & Oxystominidae & Enoplida & Enoplea \\
\hline Halalaimus sp. 4 & Oxystominidae & Enoplida & Enoplea \\
\hline Thalassoalaimus sp. 2 & Oxystominidae & Enoplida & Enoplea \\
\hline Calyptronema keiense Wieser 1953 & Enchelidiidae & Enoplida & Enoplea \\
\hline Calyptronema maxweberi (De Man, 1922) & Enchelidiidae & Enoplida & Enoplea \\
\hline Eurystomina sp. 1 & Enchelidiidae & Enoplida & Enoplea \\
\hline Abelbolla sp. 1 & Enchelidiidae & Enoplida & Enoplea \\
\hline Adoncholaimus sp. 2 & Oncholaimidae & Enoplida & Enoplea \\
\hline Oncholaimellus paracarlbergi Pastor de Ward, 1993 & Oncholaimidae & Enoplida & Enoplea \\
\hline Viscosia macramphida Chitwood, 1951 & Oncholaimidae & Enoplida & Enoplea \\
\hline Metoncholaimus sp. 1 & Oncholaimidae & Enoplida & Enoplea \\
\hline Metoncholaimus sp. 2 & Oncholaimidae & Enoplida & Enoplea \\
\hline Oncholaimus sp. 1 & Oncholaimidae & Enoplida & Enoplea \\
\hline Oncholaimus sp. 2 & Oncholaimidae & Enoplida & Enoplea \\
\hline Lauratonema sp. 1 & Lauratonematidae & Enoplida & Enoplea \\
\hline Rhabdocoma sp. 1 & Trefusiidae & Enoplida & Enoplea \\
\hline Trefusia litoralis (Allgén, 1932) & Trefusiidae & Enoplida & Enoplea \\
\hline Trefusiidae gen. 1 sp. 1 & Trefusiidae & Enoplida & Enoplea \\
\hline Bathylaimus australis Cobb, 1894 & Tripyloididae & Enoplida & Enoplea \\
\hline Tripyloides amazonicus (Gerlach, 1957) & Tripyloididae & Enoplida & Enoplea \\
\hline
\end{tabular}

Quality control description: The geo-referencing of all specimens were recorded using a Garmin eTrex Legend GPS (WGS84 Datum) with an accuracy of less than $10 \mathrm{~m}$ and with at least 5 satellites. The taxonomic identification of specimens, scientific names, and their current accurate spelling were verified by C. Pastor de Ward, a free-living marine nematode specialist. Other post-validation procedures (including geographic coordinate format, congruence between collection and identification dates, absence of ASCII anomalous characters) were checked using the Darwin Test software (http://www.gbif.es/darwin_test/Darwin_Test_in.php). 


\section{Dataset description}

Object name: Darwin Core Archive free-living marine Nematodes from San Antonio Bay (Río Negro, Argentina).

Character encoding: UTF-8

Format name: Darwin Core Archive format

Format version: 1.0

Distribution: http://ipt.cenpat-conicet.gob.ar:8081/resource?r=sao2009\#

Publication date of data: 2013-10-25

Language: English

Licenses of use: This work is licensed under a Creative Commons CC0 1.0 License http://creativecommons.org/publicdomain/zero/1.0/legalcode

\section{External datasets}

Object name: Centro Nacional Patagónico (CENPAT-CONICET)

Distribution: http://ipt.cenpat-conicet.gob.ar:8081/resource?r=sao2009\#

Object name: Ministerio de Ciencia y Tecnología de Argentina (Sistema Nacional de Datos Biológicos - SNDB)

Distribution: GBIF: http://www.gbif.org/dataset/d592283b-b00e-4a39-9499-289842ccddf1

Formatted: English (U.K.)

Field Code Changed

Metadata language: English

Date of metadata creation: $2014-08-27$

Hierarchy level: Dataset

\section{Acknowledgements}

We are grateful to Sistema Nacional de Datos Biológicos (SNDB) and Consejo Nacional de Investigaciones Científicas y Técnicas (CENPAT-CONICET) for their financial support.

\section{References}

Abebe E, Andrássy I, Traunspurger W (2006) Freshwater nematodes: ecology and taxonomy. CABI Publishing, Wallingford, United Kingdom, 752 pp.

De Ley P, Blaxter M (2004) A new system for Nematoda: combining morphological characters with molecular trees, and translating clades into ranks and taxa. Nematology Monographs and Perspectives 2: 633-653.

Lorenzen S (1994) The Phylogenetic Systematics of Free-living Nematodes. The Ray Society, Surrey, United Kingdom, 383 pp. 
Lo Russo V (2012) Comparación de comunidades de nematodos de marismas de San Antonio Oeste (Río Negro) y San Julián (Sta. Cruz). Doctoral thesis, Universidad Nacional del COMAHUE, 214 pp.

Lo Russo V, Pastor de Ward CT (2012) Neochromadora alejandroi sp. n. (Chromadorida: Chromadoridae) and Cobbia macrodentata sp. n. (Monhysterida: Xyalidae), two new species of free-living marine nematodes from the Patagonian coast. Nematology 14(7): 805-815. doi: 10.1163/145854112X627327

Lo Russo V, Villares G, Martelli A, Pastor de Ward CT, Harguinteguy C (2012) New species of Epacanthion (Nematoda: Thoracostomopsidae) from Patagonia coast, Río Negro and Chubut, Argentina. Journal of the Marine Biological Association of the United Kingdom 93: 925-934. doi: 10.1017/S002531541200080X

Lo Russo V, Villares G, Pastor de Ward CT (2015) New species of Chaetonema (Nematoda, Anoplostomatidae) and Admirandus (Nematoda, Oncholaimidae) from Patagonia, Río Negro and Santa Cruz, Argentina. Journal of the Marine Biological Association of the United Kingdom. doi: 10.1017/S0025315415002039

Pastor de Ward CT (1978) Free-living marine nematodes (Subclass Adenophorea) of the ría Deseado (Santa Cruz, Argentina). Systematic Contributions I. Annales de la Societe Royale Zoologique de Belgique 1-2: 29-45.

Pastor de Ward CT (1980) Aponema papillatum sp. nov. nueva especie de nematodo marino de Puerto Deseado, Argentina. Contribuciones Científicas del CIBIMA Nro. 160: $3-11$.

Pastor de Ward CT (1984a) Tres especies nuevas de nematodos marinos de vida libre (Chromadoridae y Comesomatidae) para la ría Deseado, Santa Cruz, Argentina. Physis A 42: 39-48.

Pastor de Ward CT (1984b) Nematodos marinos de la ría Deseado (Leptolaimina: Leptolaimidae, Haliplectidae) Santa Cruz, Argentina. I. Physis A 42: 87-92.

Pastor de Ward CT (1984c) Nematodos marinos de la ría Deseado (Monhysteroidea: Sphaerolaimidae, Monhysteridae), Santa Cruz, Argentina. 3. Contribuciones del Centro Nacional Patagónico Nro. 85: 1-15.

Pastor de Ward CT (1984d) Nematodos marinos de la ría Deseado (Axonolaimoidea: Axonolaimidae, Diplopeltidae, Comesomatidae) Santa Cruz, Argentina. 4. Contribuciones del Centro Nacional Patagónico Nro. 86: 1-21.

Pastor de Ward CT (1984e) Ptycholaimellus setosus sp. nov., nueva especie de nematodo marino de vida libre (Chromadoridae, Hypodontolaiminae) de Puerto Deseado, Santa Cruz, Argentina. Neotropica 30: 11-18.

Pastor de Ward CT (1985) Nematodos marinos de la ría Deseado (Monhysteroidea: Xyalidae), Santa Cruz, Argentina, 2. Physis A 43: 113-130.

Pastor de Ward CT (1986) Free-living marine nematodes of the Deseado river estuary (Chromadoroidea: Chromadoridae, Ethmolaimidae, Cyatholaimidae and Choniolaimidae) Santa Cruz, Argentina. 5. Publicación Especial del Centro Nacional Patagónico Nro. 6: 1-83.

Pastor de Ward CT (1988) Nematodos marinos de la ría Deseado (Desmodoroidea: Desmodoridae, Draconematidae) Santa Cruz, Argentina 7. Physis A 46: 61-72. 
Pastor de Ward CT (1989) Free-living marine nematodes of the Deseado River estuary (Siphonolaimoidea: Siphonolaimidae, Linhomoeidae) Santa Cruz, Argentina, 6. Studies on Neotropical Fauna and Environment 24: 231-247. doi: 10.1080/01650528909360794

Pastor de Ward CT (1990) Nematodos marinos de la ría Deseado (Oncholaimoidea: Oncholaimidae), Santa Cruz, Argentina, 10. Physis A 48: 29-40.

Pastor de Ward CT (1991) Nematodos marinos de la ría Deseado (Oncholaimoidea: Enchelidiidae), Santa Cruz, Argentina, 11. Physis A 49: 27-39.

Pastor de Ward CT (1991) Nematodos marinos de la ría Deseado (Microlaimoidea: Microlaimidae, Monoposthiidae), Santa Cruz, Argentina, 8. Physis A 47: 1-12.

Pastor de Ward CT (1993) Nematodos marinos de vida libre de la ría Deseado (Tripyloidina, Tripyloididae), Santa Cruz, Argentina. 13. Naturalia Patagónica 1: 61-67.

Pastor de Ward CT (1995a) Free-living marine nematodes from Deseado river estuary (Ironoidea: Leptosomatidae, Thoracostomatidae). Santa Cruz, Argentina. 12. Spixiana 18: 201-209.

Pastor de Ward CT (1995b) Nematodos marinos de la ría Deseado (Enoploidea), Santa Cruz, Argentina. 14. Physis A 50: 13-20.

Pastor de Ward CT (1996) Deontostoma species from subantarctic coasts (Nematoda, Leptosomatidae). Hidrobiologia 315: 177-187. doi: 10.1007/BF00051948

Pastor de Ward CT (1998a) Distribución espacial de nematodos libres de la ría Deseado, Santa Cruz (Patagonia, Argentina). Revista de Biología Marina y Oceanografía 33: 291311.

Pastor de Ward CT (1998b) New free-living marine nematodos from Deseado river estuary (Ironoidea: Oxystominidae) Santa Cruz, Argentina. XV. Physis A 56: 1-6.

Pastor de Ward CT (1998c) Nematodos marinos de la ría Deseado (Desmodoroidea: Desmodoridae, Draconematidae), Santa Cruz, Argentina. VII. Physis A 46: 61-72.

Pastor de Ward CT (1999) The structure of the genital apparatus in Nematoda (Chromadoroidea) from South Atlanctic Coasts. Physis A 57: 47-54.

Pastor de Ward CT, Lo Russo V (2009) Distribution of Diplolaimella and Diplolaimelloides species from Patagonian lagoons and coastal waters (Nematoda: Monhysteridae), Chubut and Santa Cruz provinces. Journal of the Marine Biological Association of the United Kingdom 89: 711-718. doi: 10.1017/S0025315409000198

Pastor de Ward CT, Lo Russo V, Villares G, Martelli A (2013) Three new species of the free-living nematode genus Oncholaimus Dujardin, 1845 (Enoplida, Oncholaimidae) from Atlantic coasts of Argentina. Journal of Morphology and Systematics (Jaen) 16: 131-141.

Platt H, Warwick RM (1983) Free-living Marine Nematodes. Part I. British Enoplids. Synopses of British Fauna (New Series) N²8, Barnes, Crothers, Cambridge University Press, 307 pp.

Platt H, Warwick RM (1988) Free-living Marine Nematodes. Part II. British Chromadorids. Synopses of British Fauna (New Series) N³8, Barnes, Crothers, Cambridge University Press, 499 pp.

Somerfield PJ, Warwick RM (1996) Meiofauna in marine pollution monitoring programmes. A laboratory manual. Ministery of Agriculture, Fisheries and Food, Directorate of Fisheries Research, Lowestoft, 71 pp.

Villares G, Pastor de Ward CT (2012) New species of Antomicron and Leptolaimus (Nematoda: Leptolaimidae) and record of Procamacolaimus (Nematoda: Camacolaimidae) from 
Patagonia coast, Chubut and Santa Cruz, Argentina. Journal of the Marine Biological Association of the United Kingdom 92: 929-939. doi: 10.1017/S0025315411000269

Villares G, Martelli A, Lo Russo V, Pastor de Ward CT (2013) Three new species and one new record of Campylaimus (Diplopeltidae, Nematoda) from Argentine coasts (Buenos Aires and Santa Cruz, Argentina). Zootaxa 3613: 083-096. doi: 10.11646/zootaxa.3613.1.4

Villares G (2014) Diversidad funcional y producción secundaria de las comunidades de nematodos de las marismas de San Antonio (Río Negro) y de la ría de San Julián (Santa Cruz). Doctoral thesis, Universidad Nacional del COMAHUE, 144 pp.

Warwick RM, Platt H, Somerfield PJ (1998) Free-living Marine Nematodes. Part III. Monhysterids. Synopses of British Fauna (New Series) N53, Barnes, Crothers, Cambridge University Press, 296 pp. 